\title{
Implementation of Machine Learning Techniques for Effective Predictive Analysis in Health Care Management
}

\author{
B.Prasad ${ }^{1}$, S Sandeep Kumar ${ }^{2}$, Ranga Jarabala ${ }^{3}$, Srilakshmi $\mathrm{CH}^{4}$ and P. Neelima ${ }^{*}$ \\ ${ }^{1}$ Department of IT, Vignan's Institute of Information Technology (A) \\ Visakhapatnam, JNTU Kakinada, Andhra Pradesh, India \\ ${ }^{2}$ Department of CSE, Koneru Lakshmaiah Education Foundation \\ (Deemed to be University), Andra Pradesh, India \\ ${ }^{3}$ Department of EEE Ramachandra College of Engineering \\ Eluru- 534007 Andra Pradesh, India \\ ${ }^{4}$ Department of IT R.M.D Engineering College, Kavaraipettai, TN, India \\ Department of Computer Science and Engineering School of Engineering and Technology \\ SPMVV University, Tirupati, Andra Pradesh, India \\ Corresponding author email: neelima.pannem@gmail.com
}

\section{ABSTRACT}

The health division has seen a huge transformation subsequent the introduction of latest computer technology $\&$ this has led to added medicinal information producing various sectors of research. In recent years, there has been surges in interest in study on assessment sustain appliances in healthcare, for example individuals relating to analysis, forecast, behavior forecast, and so on. This progress is due to increased data availability, breakthroughs in artificial intelligence and machine learning research, and contact to computational assets. Data Mining and Predictive Analysis are being used by a number of healthcare organizations. Predictive analysis utilizes assortment of statistical procedures as well as representation, machine learning \& data mining to approximate the future by breaking into past and current realism. Appropriate to the information ambitious environment of machine learning algorithms, artificial intelligence trends has achieved its full prospective when back up by large information positions. Our study examines the implications of current breakthroughs in information analytics and how these will be used to the healthcare industry, with a focus on analytical \&t prophecy applications.

\section{KEY WORDS: PREDICTIVE ANALYTICS, HEALTHCARE, MACHINE LEARNING, LEARNING ALGORITHMS.}

\section{INTRODUCTION}

During rising national healthcare costs, healthcare companies need relationships dynamically to discover ways to lower costs without jeopardizing patient results. One of the strategies to achieve these goals is by focusing

Biosc Biotech Res Comm P-ISSN: 0974-6455 E-ISSN: 2321-4007

\section{crossef}

Identifiers and Pagination

Year: 2021 Vol: 14 No (7) Special Issue

Pages: 205-210

This is an open access article under Creative

Commons License Attribn 4.0 Intl (CC-BY).
DOI: $h t t p: / / d x$.doi.org/10.21786/bbrc/14.7.48 on patients who are likely to be readmitted to treatment, given that these individuals are a huge amount of administrative costs (Cucciare and 0'Donohue, 2006). Government Accountability Office stated that 5 percent of Medicaid beneficiaries accounted for half of programme expenses (Quinn et al 2016). These patients generally face complex, permanent problems that are difficult to assess and control. If these patients are differentiated by healthcare providers, the latter can possibly reduce costs and help them manage their ailments better.

This research examines and perhaps benefits a strategy for distinguishing patients with and without the danger of readmission. These patients are supplied with solutions tailored to their specific needs and can be utilized to
Article Information

Received: $13^{\text {th }}$ May 2021

Accepted after revision: $19^{\text {th }}$ July 2021 
plan improved solutions for similar patients using the data obtained from these patients. As customers become constantly aware of the adaptation of their insurance buys to their unique demands, the top health care provider has started evaluating how machine learning (ML) can improve business and buyer loyalty. The classification of data with machine learning algorithms will effectively collect billions of their current items (Pandey et al 2017).

Healthcare is likely more important to anticipate than explain given the tremendous expense of interruption in analysis and behavior (Agarwal and Dhar 2014). The significance of prognostic analytics in healthcare has been emphasized frequently in previous IS research. The digitalization of health care creates vast novel information sets in the health sector. Prospective clinical information resources include electronic entries for medical practitioners, medical clarification, $\mathcal{C}$ imaging equipment, presently to list them. These are extremely complicated and fragmented as compared with remaining businesses (Raghupathi and Raghupathi 2014), which pose enormous diagnosis, treatment and preventive issues and are of incalculable importance for improvement.

Predictive analysis enables the life sciences and healthcare providers and uses numerous approaches as of data mining, machine learning and artificial intelligence to examine present findings to forecast the expectations. It helps healthcare companies plan for healthcare by improving costs (Balaji et al 2021), effectively diagnosing diseases, and improving patient care, managing resources and improving clinical result. Deep learning is a concept for digging massive volumes of information to routinely detect models \& mine characteristics from composite uncontrolled information lacking individual participation. In diagnostic applications, machine learning plays a vital role (Sreedhar, et al 2020). Machine learning methods can expose significant data included in vast information to support clinical decision making, and then provide clinicians with correct analysis of each condition for improved therapy, resulting in better medical choices.

2. Related Works: Machine learning plays a key role in the healthcare profession. Many systems help to cope with and understand the use of large data to care for well-being and medical data (Nambiar et al 2013). Machine learning can change the healthcare industry into a modernization of operational skills, help forecast and develop numerous diseases, enhance clinical preliminary checks and rationalize insurance expenditures at all levels (Yoo, et al 2012). Authors have provided an overview of several data mining approaches utilized in the biological and health insurance fields (Malchi et al 2021). Predictive analysis is an engaging big data specialist and identifies important designs to estimate the future opportunity. It helps to enhance business or promote assets in different areas of this field. It has been found that fresh and substantial meetings made through the research of medical information can make generous pledges to research technology, treatment selection and omen predictions (Hachesu, et al 2013). Expected results, such as readmission, the beginning of specific disease and the length of stay (LoS) are the most absolute predictive outcomes (Gampala, et al 2020 and Natarajan et al 2020).

Data preprocessing, alteration have been utilized (Peneti, et al 2021) to generate knowledge regarding the interplay between several measures and patient survival. In the variety of resolution policies, 02 split information removal methods were utilized to extract knowledge. Such regulations have been utilized by a choice production algorithm predicting new unseen patients' survival. Important data mining parameters were interpreted for their medical importance. In their research, they presented an idea and evaluated it by means of composed information from 04 dialysis facilities. This method shown in their study decreases the price \&t labour of choosing people for experimental trials. Patients will be selected depended on forecast outcomes \& the key restrictions.(Kara et al 2006).

Utilized two procedures in the neural organization, the Back Propagation Algorithm (BPA), the Radial Basic Function(RBF) and one nonlinear Vector Support Machine (SVM) characterization and contrasted it in agreement and their productivity and exactness. They utilized WEKA 3.6.5 instrument to choose the best innovation among the three Kidney Stone Diagnostic Algorithms referenced. Their proposition predominantly pointed toward proposing the best clinical symptomatic instrument, for example, the recognizable proof of kidney stones, to lessen finding time and increment proficiency and precision. Back proliferation (BPA) further developed extraordinarily the standard arrangement procedures for clinical use from the test information finished up.

The Pre-Processing and data transformation methods of (Malchi et al 2021) were utilized to generate awareness concerning the connection among many of the deliberate characteristics \&t patient endurance. 02 separate information mining procedures were worn in the variety of result policies to dig out information. These regulations have been used by a decision-making algorithm predicting new unseen patients' survival. Important data mining parameters were interpreted for their medical importance. In their research work, they proposed a new concept, were pertained \&t experienced utilizing acquired information in 04 dialysis locations. The approach proposed in their research lowered patient 
selection costs and efforts for clinical investigations. Patients can be selected depending on the findings of the forecast and the most significant parameters.

In a paper on the use of machine learning techniques for example SVM \&t Random Forest (Agarwal et al 2016). These were used to examine, classify, and compare information sets with various kernels \& its constraint for malignancy, liver, and heart illness. RF Results and SVM are evaluated for several information sets such as data set for cancer, liver infection dataset and cardiovascular data set. The outcomes of various elements were customized to the correct selection of parameters. The results were properly examined to develop better prediction learning systems. It is concluded that with the SVM classification approach with different kernel functions varied outcomes have been seen. (Ganesh, et al 2020) Centered the investigation of example electro retinografy (PERG) information utilizing counterfeit neural organization to analyze optic nerve disease (ANN). Carried out Multilayer feed ANN prepared with a back engendering approach for Levenberg Marquart (LM). The end-product were classed as sound and sick. The outcomes showed that the proposed PERG approach might be deciphered viably.

Proposed System: The proposed system is divided into several layers, including client, information, dispensation, and representation. A modular planning permits the analytical basis to implement new features lacking rewriting the top layers. In the current concept system proof, the analytics envisaged are predictive and allow neural networks to be trained graphically and to use the model in place to predict values for fresh input.

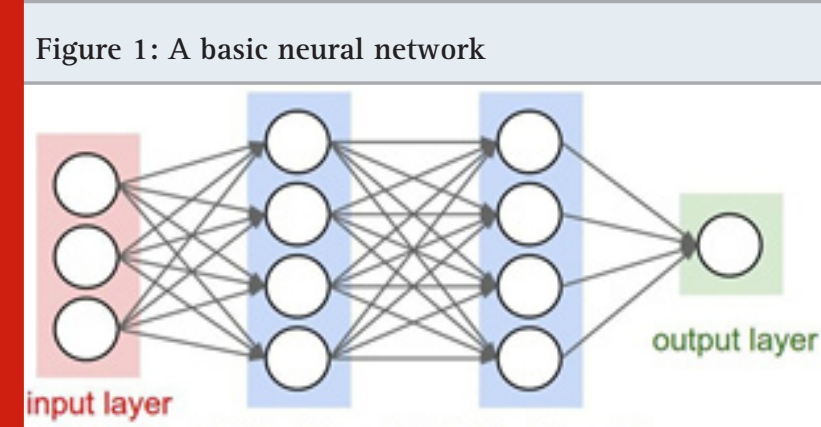

hidden layer 1 hidden layer 2

Figure 2: System flow architecture

\section{Predictive Analytics Process}

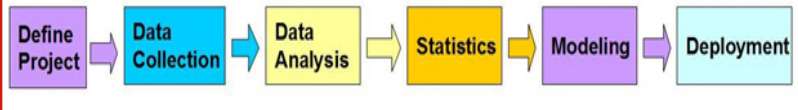

The User Tier: In addition to organizing datasets, it provides a way for researchers to execute visualizations and analytics on any dataset they choose. It serves as a boundary to the information layer, where statistical sets were maintained in their honor and are stored strongly. The Cassandra clustered database system is used in this cloud-based storage strategy, which provides capabilities like as more usage, error patience, and multiple information core duplication (Rakshit, S., et al 2017), among others. Safety of the connection is critical in order to avoid information stealing while it is being transmitted both within and external of the cloud storeroom system.

Preparing Tier: In particular, it is at this layer that the real investigation is performed. For preparing, the information is recovered from the layer above, and the model is fitted to the dataset utilizing the boundaries got from the client level, which are gotten from the client level. It then, at that point stores the model in the last level, which is alluded to as the model level; recently prepared models are recovered from this layer in order to conduct predictive queries on the results. Another procedure that may be performed is the loading of weights onto an existing model, which will enable for the training of an older model to be extended further.

Figure 3: Flow diagram for predictive analytics

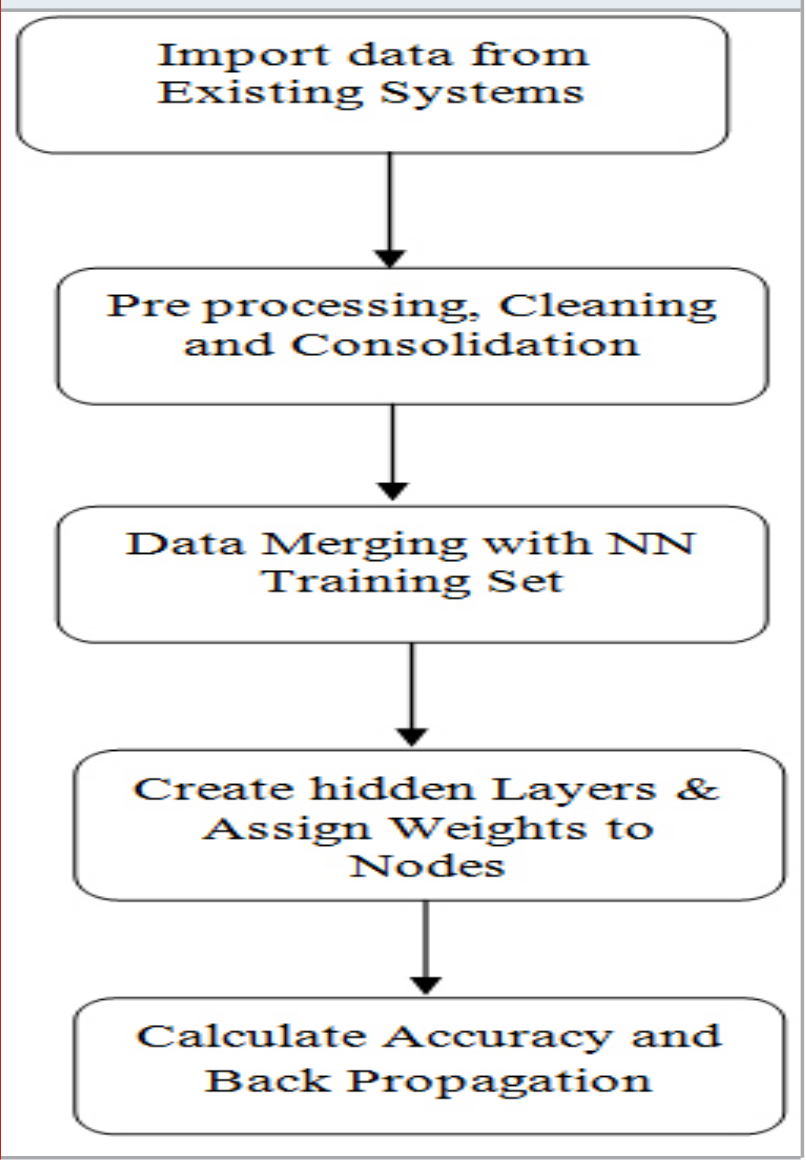

Model Tier: The model level is liable for putting away the neural organization objects in a spellbinding arrangement, just as the loads of the neurons that are associated with the items in the model. It is profoundly 
incorporated with the handling level because of the absence of normal guidelines among various neural organization structures in the business.

3.1 Secondary systems: It also has a feature for integrating through third-party method and allowing for data significance functionality, among other things. However, due to the absence of readily accessible systems at the moment, this was limited to a abstract point only thus far.

Figure 2 portrays an ordinary System stream model for a situation in which the model is to be utilized. A UI is accommodated bringing in existing datasets in normalized organizations like CSV, which are exposed to both manual and mechanized second looks for good measure. This data is consequently saved inside in a construction that is agreeable with the NeHA (National e-Health Authority) designs, which are itself gotten from the most broadly, utilized universally perceived principles. In the following step, the investigator has the option of manually merging reports with an accessible neural network depended on shared constraints, or starting again from the beginning. Now that the neural network has been trained using the dataset that has been collected, it may be used for predictive analytics purposes.

Predictive and statistical functions can be applied to the datasets in order to create high-level visualizations, which can then be used by academics and other healthcare professionals to make better decisions. If geo-tagged fitness reports are routinely collected by area, they can reveal several current styles in the field, for example influx of a exacting transferable virus or the presence of toxins from diverse sources, among other things. These can assist the necessary authorities in taking timely corrective actions, or even preventative procedures, before the incident of a problem occurring. These advantages can be obtained through efficient integration with secondary reporting systems as well as by widespread application of the system (Kumar, M.S. and Prakash, K.J., 2019).

Figure. 3 show the entire progression of the prescient investigation measure. The information from an assortment of heterogeneous sources is brought into the framework in the initial step. There are numerous unmistakable kinds of data sets, information shops, and capacity stockrooms, all with a wide range of structures and designs. The subsequent stage is to preinteraction and cleans the information to blend it with the framework and to guarantee that it is reliable with the National Healthcare Act rules. Pre-preparing wipes out or revises any tuples that contain botches, absent or wrong qualities, exceptions, or different inconsistencies. When the information has been tidied and tidied up to the satisfactory guidelines, it is fit to be joined with the current information and utilized as a component of the neural organization preparing set (s). The neural organization then recomputes the loads for the hubs in the secret layers to represent the new information that has been taken care of to the framework. Back engendering is directed in the last stage, utilizing the re-processed hubs, and the exactness of the neural organization is improved thus.

4. Simulation Results: The National Institute of Diabetes and Digestive and Kidney Diseases (NIDDK) is the organization that originally created the dataset. The goal is to forecast whether one patient has diabetes or not depending on analytical readings while imposing a large number of constraints on the main database. The dataset contains, in particular, all records of female patients aged 25 or older of Pima Indian ancestry (Lohr, S., 2016), with no records of male patients.

\section{Parameters}

- Pregnancies: Number of times pregnant

- Glucose: Plasma glucose focus a 2 hour

- Blood Pressure: Diastolic pulse (mm Hg)

- Skin Thickness: Triceps skin crease thickness (mm)

- Insulin: 2-Hour serum insulin (mu U/ml)

- BMI: Body mass list (weight in $\mathrm{kg} /($ tallness in $\mathrm{m}) 2$ )

- Diabetes Pedigree Function: Diabetes pedigree work

- Age: Age (a long time)

- Outcome: Class variable (2/3)

Table 1. Simulation Results

\begin{tabular}{|c|c|c|c|c|}
\hline \multicolumn{3}{|c|}{ Layers } & \multirow{2}{*}{ Epochs } & Accuracy (\%) \\
\cline { 1 - 2 } First & Second & Third & & \\
\hline 15 & 10 & 2 & 50 & 81.35 \\
\hline 15 & 10 & 2 & 100 & 83.68 \\
\hline 15 & 10 & 2 & 150 & 85.68 \\
\hline 30 & 20 & 2 & 50 & 87.29 \\
\hline 30 & 20 & 2 & 100 & 88.56 \\
\hline 30 & 20 & 2 & 150 & 89.36 \\
\hline 60 & 40 & 2 & 50 & 85.26 \\
\hline 60 & 40 & 2 & 100 & 92.56 \\
\hline 60 & 40 & 2 & 150 & 97.56 \\
\hline
\end{tabular}

Table 1 depicts a table showing the impact of changing the quantity of nodes available in each layer of the neural network (there are three levels). As demonstrated above, the precision of predictions as the quantity of times (a number of surpasses) rises, The quantity of layers nodes. Figure 4 shows permanent data from the dataset organized in ordered columns according to their respective values. The Y- axis is the occurrence of every type of things. The 09 histograms in Figure 4 show the uniqueness $\&$ the value allotment of the 09 
restrictions stated above. The client may quickly view the information set and comprehend the values and nature of the dataset.

Figure 4: Visualization

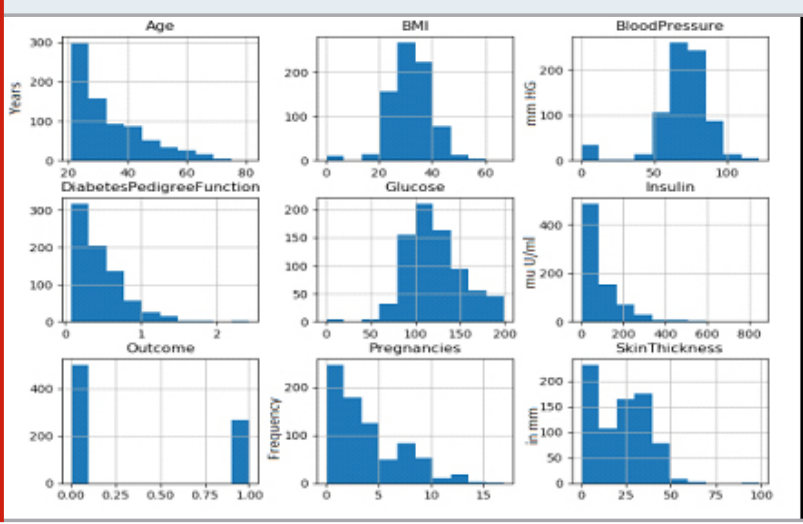

Future Trends of Deep Learning in Healthcare Predictions:

Scientists and merchants in the clinical area trust in profound learning applications that take on people and not exclusively will insightful apparatus be utilized for diagnostics yet additionally assists meds with being endorsed, infection anticipated and treatment directed in future. Since the presentation of advanced imaging, profound learning procedures applied to clinical imaging are self-evident. Google Deep Mind Health (Natarajan, V.A., et al 2020) works with the United Kingdom's National Health Service to handle the clinical information of more patients in the five emergency clinics. IBM Watson has as of late extended its imaging business by buying Merge's clinical administration stage covering radiology, cardiology, muscular health, eye care and other clinical claims to fame by bringing billions of dollars into the imaging field. Reports that they utilize significant learning methods to gather billions of clinical instances of bits of knowledge and make specialists quicker and more exact. Uses a profound learning strategy and gives guardians to screen youngsters' wellbeing access continuous through a smart device, limiting regular doctor visits.

AI will be utilized to blend visual information and engine designs in gadgets like the da Vinci to make it workable for machines to direct future activities. Profound learning in the field of information examination in clinical imaging is a developing pattern, in spite of the fact that there are still deterrents in this field. Applying profound learning calculations to clinical symbolism is an interesting and extending subject of exploration. Informational collection inaccessibility, given doctors, standard information AI strategies, protection, and legitimate troubles are the difficulties. Customized medication remembers ideas for medical services and sickness treatments dependent on clinical history, hereditary parentage, past afflictions, food, feelings of anxiety, and so forth

\section{CONCLUSION}

In this article, we presented a short summary of machine learning research as regards predictive analysis of health data and its motivation in healthcare. Many efforts have been made to minimize the cost of treatment while simultaneously improving the quality of care. There is a fundamental necessity for procedures that can contribute to the transformation of a healthcare organization. For the purposes of finale, the usage of data mining methods for analytical study was extremely significant in the health field since it provides us with the ability to confront virus that intimidate individuals of all ages; children, young children, and elderly citizens, during the expectation of treatment and assistance in decisionmaking, earlier in their development. Subsequently, the model satisfies its definitive motivation behind giving a financially savvy stage to clinical datasets to be utilized with regards to prescient just as broad examination.

It is currently being assessed with an assortment of datasets to broaden its application scope significantly further and to guarantee that it's anything but confined to a specific dataset.

\section{REFERENCES}

Agarwal, R. and Dhar, V., 2014. Big data, data science, and analytics: The opportunity and challenge for IS research.

Agarwal, V., Zhang, L., Zhu, J., Fang, S., Cheng, T., Hong, C. and Shah, N.H., 2016. Impact of predicting health care utilization via web search behavior: a data-driven analysis. Journal of medical Internet research, 18(9), p.e251.

Balaji, K., Kiran, P.S. and Kumar, M.S., 2021. An energy efficient load balancing on cloud computing using adaptive cat swarm optimization. Materials Today: Proceedings.

Cucciare, M.A. and O’Donohue, W., 2006. Predicting future healthcare costs: how well does risk-adjustment work? Journal of health organization and management.

Gampala, V., Kumar, M.S., Sushama, C. and Raj, E.F.I., 2020. Deep learning based image processing approaches for image deblurring. Materials Today: Proceedings.

Ganesh, D., Kumar, T.P. and Kumar, M.S., 2020. A Dynamic and adaptive learning mechanism to reduce cross layer attacks in cogntive networks. Materials Today: Proceedings..

Garikapati, P., Balamurugan, K., Latchoumi, T.P. and Malkapuram, R., 2021. A Cluster-Profile Comparative Study on Machining AlSi 7/63\% of SiC Hybrid Composite Using Agglomerative Hierarchical Clustering and K-Means. Silicon, 13, pp.961-972. 
Hachesu, P.R., Ahmadi, M., Alizadeh, S. and Sadoughi, F., 2013. Use of data mining techniques to determine and predict length of stay of cardiac patients. Healthcare informatics research, 19(2), pp.121-129.

Kara, S., Güven, A. and Öner, A.Ö., 2006. Utilization of artificial neural networks in the diagnosis of optic nerve diseases. Computers in Biology and Medicine, 36(4), pp.428-437.

Kumar, M.S. and Prakash, K.J., 2019. Internet of things: IETF protocols, algorithms and applications. Int. J. Innov. Technol. Explor. Eng, 8(11), pp.2853-2857.

Lohr, S., 2016. IBM is counting on its bet on Watson, and paying big money for it. New York Times, 17(10), p.2016.

Malchi, S.K., Kallam, S., Al-Turjman, F. and Patan, R., 2021. A trust-based fuzzy neural network for smart data fusion in internet of things. Computers \& Electrical Engineering, 89, p.106901.

Nambiar, R., Bhardwaj, R., Sethi, A. and Vargheese, R., 2013, October. A look at challenges and opportunities of big data analytics in healthcare. In 2013 IEEE international conference on Big Data (pp. 17-22). IEEE.

Natarajan, V.A., Kumar, M.S., Patan, R., Kallam, S. and Mohamed, M.Y.N., 2020, September. Segmentation of Nuclei in Histopathology images using Fully Convolutional Deep Neural Architecture. In 2020 International Conference on Computing and Information Technology (ICCIT-1441) (pp. 1-7). IEEE.

Natarajan, V.A., Babitha, M.M. and Kumar, M.S., 2020. Detection of disease in tomato plant using Deep Learning Techniques. International Journal of Modern Agriculture, 9(4), pp.525-540.

Pandey, S., Supriya, M. and Shrivastava, A., 2017, September. Data classification using machine learning approach. In The International Symposium on Intelligent Systems Technologies and Applications (pp. 112-122). Springer, Cham.

Peneti, S., Kumar, M.S., Kallam, S., Patan, R., Bhaskar, V. and Ramachandran, M., 2021. BDN-GWMNN: Internet of Things (IoT) Enabled Secure Smart City Applications. Wireless Personal Communications, pp.1-17.

Prasath, S., Validating Data Integrity in Steganographed Images using Embedded Checksum Technique. International Journal of Computer Applications, 975, p.8887.

Quinn, K., Weimar, D., Gray, J. and Davies, B., 2016. Thinking about clinical outcomes in Medicaid. The Journal of ambulatory care management, 39(2), p.125.

Raghupathi, W. and Raghupathi, V., 2014. Big data analytics in healthcare: promise and potential. Health information science and systems, 2(1), pp.1-10.

Rakshit, S., Manna, S., Biswas, S., Kundu, R., Gupta, P., Maitra, S. and Barman, S., 2017, March. Prediction of diabetes type-II using a two-class neural network. In International Conference on Computational Intelligence, Communications, and Business Analytics (pp. 65-71). Springer, Singapore.

Sreedhar, B., BE, M.S. and Kumar, M.S., 2020, October. A Comparative Study of Melanoma Skin Cancer Detection in Traditional and Current Image Processing Techniques. In 2020 Fourth International Conference on I-SMAC (IoT in Social, Mobile, Analytics and Cloud)(I-SMAC) (pp. 654-658). IEEE.

Yoo, I., Alafaireet, P., Marinov, M., Pena-Hernandez, K., Gopidi, R., Chang, J.F. and Hua, L., 2012. Data mining in healthcare and biomedicine: a survey of the literature. Journal of medical systems, 36(4), pp.2431-2448. 